\title{
The COMPASS future: COMPASS II
}

\author{
E. Rocco, ${ }^{1}$ \\ on behalf of the COMPASS collaboration \\ ${ }^{1} \mathrm{CERN}$, Geneva, Switzerland
}

December 8, 2011

\begin{abstract}
Up to now the COMPASS experiment [1] contributed with the muon beam to precise measurements of the nucleon longitudinal momentum structure. A more complete nucleon description beyond collinear approximation includes the quark intrinsic momentum distributions which are described by the Transverse Momentum Dependent (TMD) Parton Distribution Functions (PDFs) and by Generalised Parton Distributions (GPDs). The next phase COMPASS II [2] approved in December 2010 focuses on GPDs measurements through Deep Virtual Compton Scattering (DVCS) and Deep Virtual Meson Production (DVMP), in TMDs with Drell-Yan process and on Primakoff measurements. The latter shed light on the hadron structure through the hadron deformation in an external electromagnetic field described by the polarisabilities.
\end{abstract}

\section{Introduction}

COMPASS is a fixed target experiment at CERN using the unique CERN SPS M2 beamline that is able to deliver high energy hadrons and polarised muons beams within the energy range of 50 to $280 \mathrm{GeV}$. Its forward spectrometer extends along 50 meters with two dipole magnets, more than 300 tracking planes and particle identification capabilities by a Ring Imaging CHerenkov (RICH) detector and two sets of electromagnetic and hadronic calorimeters. The target can be polarised longitudinally or transversely with respect to the beam direction, depending on the specific physics program.

Since 2002 COMPASS is contributing to the description of the nucleon structure and the future physics program is going to improve this description through two complementary approaches. The extracted GPDs through the DVCS and DVMP measurements, provide a three dimensional description of the nucleon [4] (nucleon tomography) correlating fraction of longitudinal momentum of nucleon carried by the quarks with the spatial distribution in the transverse plane. On the other hand eight TMDs relating $x_{B j}$ to $k_{T}$ are provided to get the complementary three dimensional description [5]. The DY measurements give access to the azimuthal asymmetries in the valence quark region where the theory predicts sizable asymmetry.

The COMPASS phase II also comprises an accurate and precise measurement of the pion polarisabilities through the Primakoff process and the first measurement of the kaon polarisability. 


\section{Deep Virtual Compton Scattering}

GPDs are experimentally accessible in exclusive reactions such as DVCS and Deeply Virtual Meson Production (DVMP). In the COMPASS kinematic domain the GPD $H$ yields the dominant contribution in measurements with an unpolarised target, while GPD $H$ and $E$ are accessible with a transversely polarised target. They depend on the photon virtuality $Q^{2}$, the total four-momentum transfer squared $t$ between the initial and final nucleon states and two additional variables $x$ and $\xi$ representing the average and half the difference between the initial and final longitudinal momentum fraction of the nucleon carried by the parton (see Fig. 1). In DVCS and DVMP processes, $x$ is an internal variable while the skewness $\xi$ is related to the Bjorken variable $x_{B j}=\frac{Q^{2}}{2 M \nu}$ in the Bjorken limit: $\xi=\frac{x_{B j}}{\left(2-x_{B j}\right)}$.

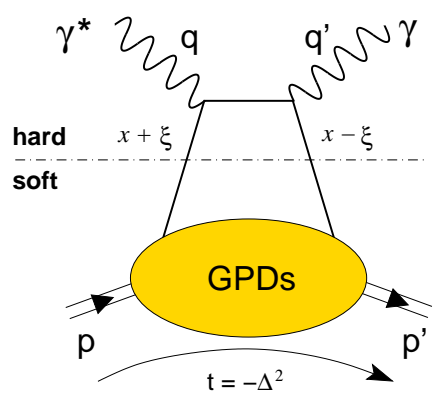

Figure 1: Handbag diagram for the DVCS process at the leading twist.

DVCS is considered as the cleanest of the experimentally accessible processes because the effects of the next-to-leading order and subleading twists are under theoretical control [3]. A competing process is the Bethe-Heitler $(\mathrm{BH})$ process of elastic lepton-nucleon scattering with a hard Bremsstrahlung photon emitted by the incoming or outgoing lepton. It produces the same final state as DVCS so that both processes interfere at the level of amplitudes, $\mathscr{A}$ :

$$
d \sigma(\mu N \rightarrow \mu N \gamma) \propto\left|\mathscr{A}_{B H}\right|^{2}+\left|\mathscr{A}_{D V C S}\right|^{2}+\mathscr{A}_{B H} \mathscr{A}_{D V C S}^{*}+\mathscr{A}_{B H}^{*} \mathscr{A}_{D V C S}
$$

In the COMPASS experiment, kinematics domains are explorable where either BH or DVCS dominates. The collection of almost pure BH events at small $x$ allows one to get an excellent reference yield and to control accurately the efficiency of the detection. On the contrary, the collection of almost pure DVCS at larger $x$ will allow for the measurement of the $x$-dependence of the $t$-slope of the cross section which is related to the tomographic partonic image of the nucleon.

The dependence on $\phi$, the azimuthal angle between the lepton scattering plane and the photon production plane, is a characteristic feature of the cross section. Integration over $\phi$ and/or analysis of the angular dependence in $\phi$ allows to isolate specific contributions that are sensitive to different combinations of quarks GPDs.

COMPASS is presently the only facility to provide polarised leptons with both charges: polarised $\mu^{+}$and $\mu^{-}$beams. It should be noted that for muon beams the charge and polarisation are naturally reversed simultaneously. This feature allows with the same apparatus the measurement of the beam charge $(\mathrm{C})$ and spin $(\mathrm{S})$ sum $\mathscr{S}$ and difference $\mathscr{D}$ :

$$
\mathscr{S}_{C S, U} \equiv d \sigma^{+\downarrow}+d \sigma^{-\uparrow}, \mathscr{D}_{C S, U} \equiv d \sigma^{+\downarrow}-d \sigma^{-\uparrow},
$$

where the arrow indicates the beam polarisation and $U$ the unpolarised target condition. From these measurements the real and imaginary part of Compton Form Factors (CFF) can be extracted. A $\mathrm{CFF}$ is a sum over flavors $f$ of convolutions of respective GPDs with a perturbatively calculable kernel describing the hard $\gamma^{*} q$ interaction. In the difference $\mathscr{D}_{C S, U}$ the pure $\mathrm{BH}$ cancels out and the analysis of 
the $\phi$ dependence will provide a measurement of the real part of the corresponding CFF. The projected accuracy for the $\phi$ dependence of the beam charge and spin asymmetry is shown in Fig. 2 .

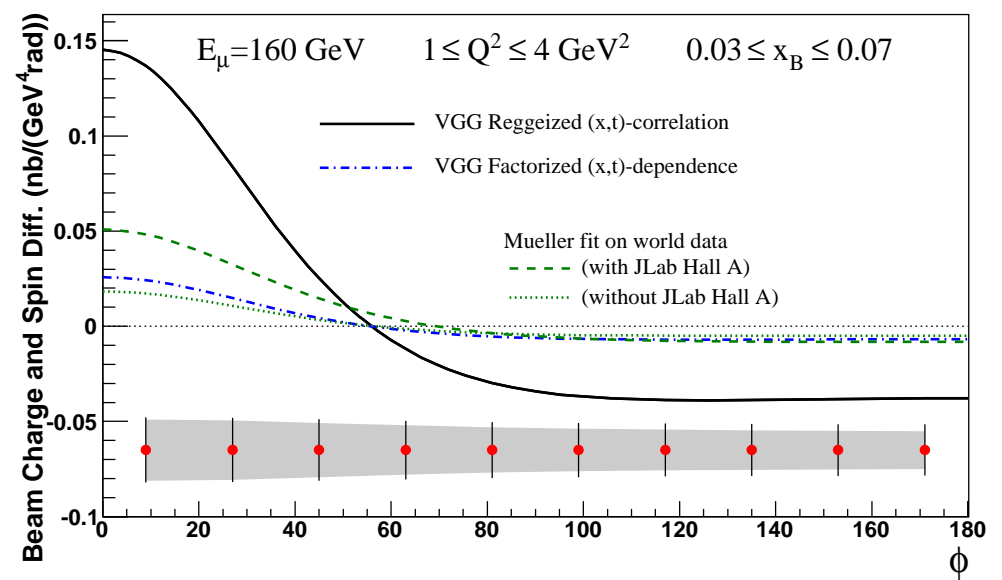

Figure 2: Projected statistical accuracy for a measurement of the $\phi$ dependence of the beam charge and spin asymmetry. Predictions are calculated using the VGG model [6]. The green curves show predictions based on the first fit of the world data [7].

In the $\mathscr{S}_{C S, U}$ the BH doesn't cancel out and it has to be subtracted. Integrating over $\phi$ yields the $t$-dependence of the cross section which is related to the transverse size of the nucleon at different values of $x_{B j}$. In the simple ansatz

$$
\frac{d \sigma\left(x_{B j}\right)}{d t} \sim \exp \left(-B\left(x_{B j}\right)\right)|t|
$$

and

$$
B\left(x_{B j}\right)=B_{0}+2 \alpha^{\prime} \log \left(\frac{x_{0}}{x_{B j}}\right)
$$

the shrinkage parameter $\alpha^{\prime}$ is used to describe the decrease of the nucleon size with the increasing $x_{B j}$.

In 2008 and 2009 two test measurements were performed with a $40 \mathrm{~cm}$ long liquid hydrogen target using a recoil proton detector for the TOF, energy loss measurements and triggering purposes. These results have confirmed the feasibility of the future DVCS measurements. Figure 3 represents the data collected during the 2009 beam test.

In order to perform the DVCS measurement new equipment and a spectrometer upgrade are foreseen. A 4 meter long Recoil Proton Detector (RPD) housing the 2,5 meter long hydrogen target will be built together with a new electromagnetic calorimeter, called ECAL0, positioned right behind the RPD to guarantee a larger photon angles coverage. These new detectors are the major projects. For a complete and comprehensive description and explanation of the spectrometer upgrade, we refer to Ref. [2].

\section{Drell-Yan measurements}

A complementary description of the nucleon structure is provided by the TMDs. They supply a dynamic picture by taking into account the intrinsic transverse momentum $k_{T}$ of its constituents. In the first phase of COMPASS the Sivers TMD and the Boer-Mulders TMD were measured off transversely polarised targets and the TMDs of these measurements were convoluted with fragmentation functions. In the Drell-Yan process (Fig. 4), an alternative approach is provided because the fragmentation functions are not anymore involved, but only a convolution of the target and the projectile TMDs. Such 

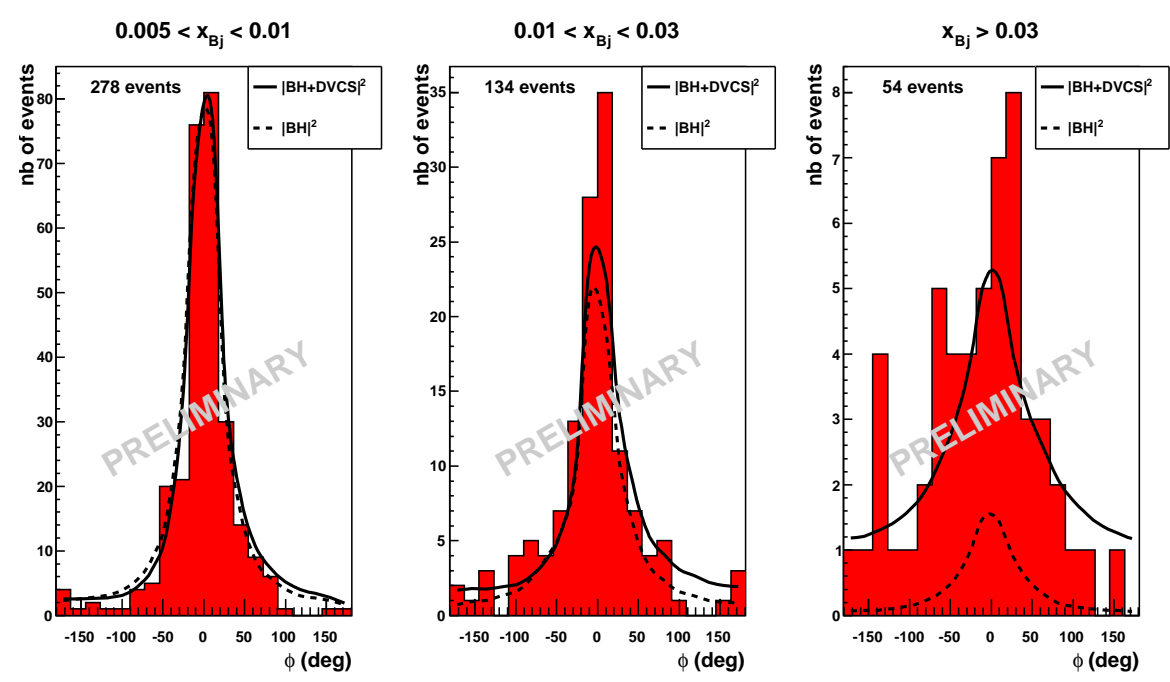

Figure 3: Distribution in the azimuthal angle $\phi$ for measured exclusive single photon events, $\mu p \rightarrow \mu^{\prime} p \gamma$ with $Q^{2}>1 \mathrm{GeV}^{2}$.

data will allow for a test of the factorisation ansatz: the sign of the Sivers and Boer-Mulders functions are expected to be opposite in DY and SIDIS. Moreover other azimuthal asymmetries as pretzelosity and transversity can be studied in the valence quark region where theory predicts sizable contributions.

Figure 5 shows the theoretical predictions and the expected statistical and systematic errors for the Sivers asymmetry.

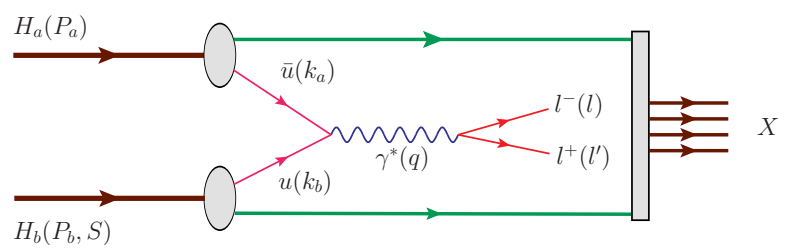

Figure 4: Drell-Yan Feynman diagram: the annihilation of a quark-antiquark pair into a lepton pair.

The Drell-Yan measurement will be performed with a $\pi^{-}$beam at $190 \mathrm{GeV} / \mathrm{c}$ and on the transversely polarised $\mathrm{NH}_{3}$ target with two cells. A long hadron absorber located downstream the target will reduce the background of produced hadrons (pions and muons which may decay to muons). A tungsten plug along the beam axis will stop the non-interacting beam. A di-muon trigger based on large acceptance hodoscopes will select the interesting events. The dominant process is the annihilation of the valence anti-quarks from the pion (beam) with a valence quark from the proton (target). The combinatorial background can be estimated using like-sign muon pairs. Muons pairs in the mass range of $4 \leq \mathrm{M}_{\mu \mu} /$ $\mathrm{MeV} / \mathrm{c}^{2} \leq 9$ will be used to extract the signal as the estimated background is very small in this region.

In 2007, 2008 and 2009 short beam tests were performed to verify the feasibility of DY program and to evaluate in realistic way the achievable statistical accuracies.

The main goals of the 2007 beam test were to measure the radiation conditions inside the experimental hall and the rise of the polarised target temperature when using an incident beam as well as to observe the behaviour of the whole COMPASS spectrometer. The high intensity hadron beam causes multiple nuclear interactions in the target with production of a large amount of secondary particles. This contributes to an increase of the target temperature and shortens the spin relaxation time of the 


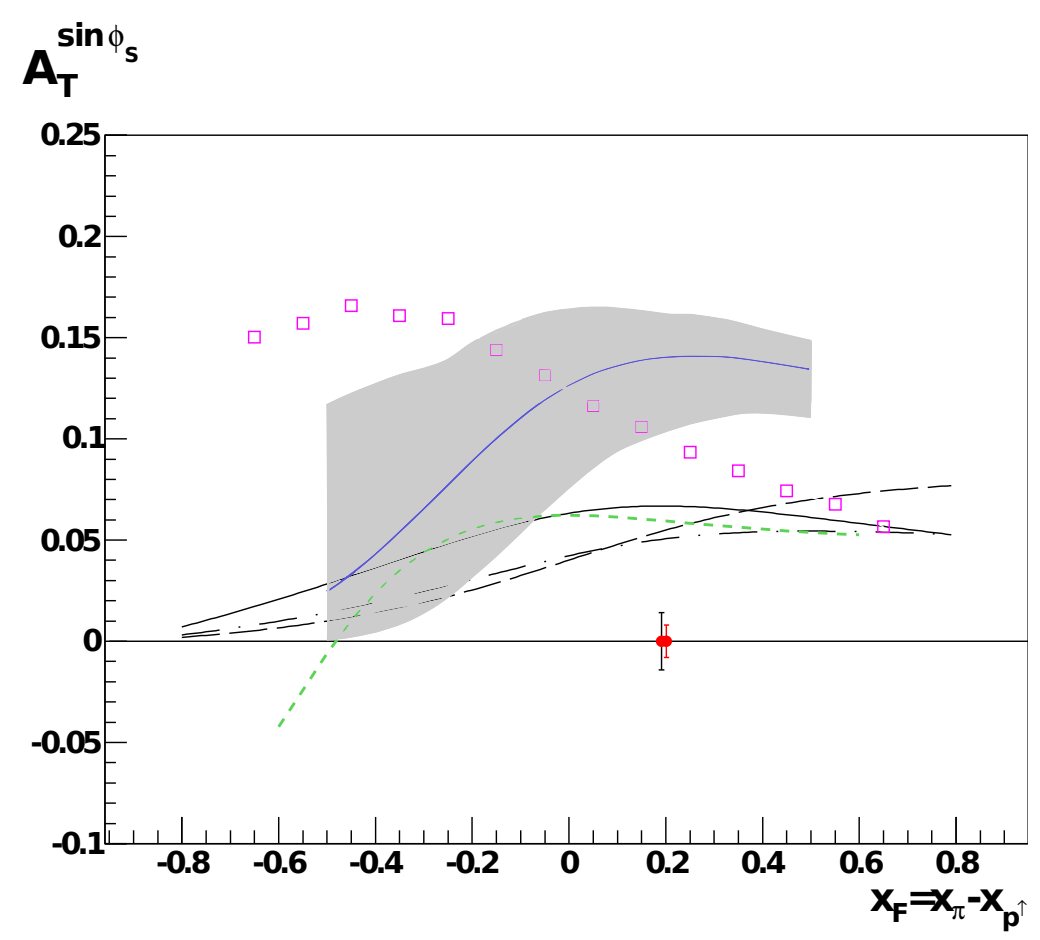

Figure 5: Theoretical predictions and expected statistical (left) and systematic (right) error for a measurement of the Sivers asymmetry in the high mass region $4 \leq \mathrm{M}_{\mu \mu} / \mathrm{MeV} / \mathrm{c}^{2} \leq 9$.

material. In 2008 the energy beam was increased compared to the previous test from $160 \mathrm{GeV}$ till 190 $\mathrm{GeV}$. The attention was focused on the detectors occupancy and the DAQ capability to handle such a high data rate. The outcome of these two short beam tests was the absolute need of the hadron absorber placed right downstream the target. The disadvantage of this solution is the limitation to di-muons in the final states.

In 2009 beam test the COMPASS spectrometer was equipped with a hadron absorber in order to suppress the flux of secondary hadrons produced in the interactions of the $\pi^{-}$beam at $190 \mathrm{GeV}$ with the dummy target made of polyethylene. In the centre of the absorber, along the beam axis a beam plug made of tungsten was installed in order to stop the non-interacting beam particles. The number of found $J / \psi$ 's was in good agreement with the expected $J / \psi$ yield as also the number of the DY events in the range of $4 \leq \mathrm{M}_{\mu \mu} / \mathrm{MeV} / \mathrm{c}^{2} \leq 9$ was, (see Fig. 6).

As for the GPD physics program new equipment will be build. The hadron absorber, the beam plug are the essential components to be implemented into the spectrometer. For further details of the DY experimental setup, we refer to Ref.[2].

\section{Primakoff process}

In understanding the strong interactions, the pion and its properties played a key role, first as an exchange particle in the nucleon-nucleon interaction and later as the lightest quark-antiquark bound state of QCD. So far the pion's global properties and the details of its internal structure are still a challenge for the experimental and theoretical physics in verifying QCD for non-perturbative bound states and low-energy reactions.

The Chiral Perturbation Theory (ChPT) is a low-energy effective field theory based on the approximate chiral symmetry of the QCD Hamiltonian, possible for small quark masses as $u, d$ and $s$. The crucial feature of the ChPT is to establish various connections between physical observables as a 


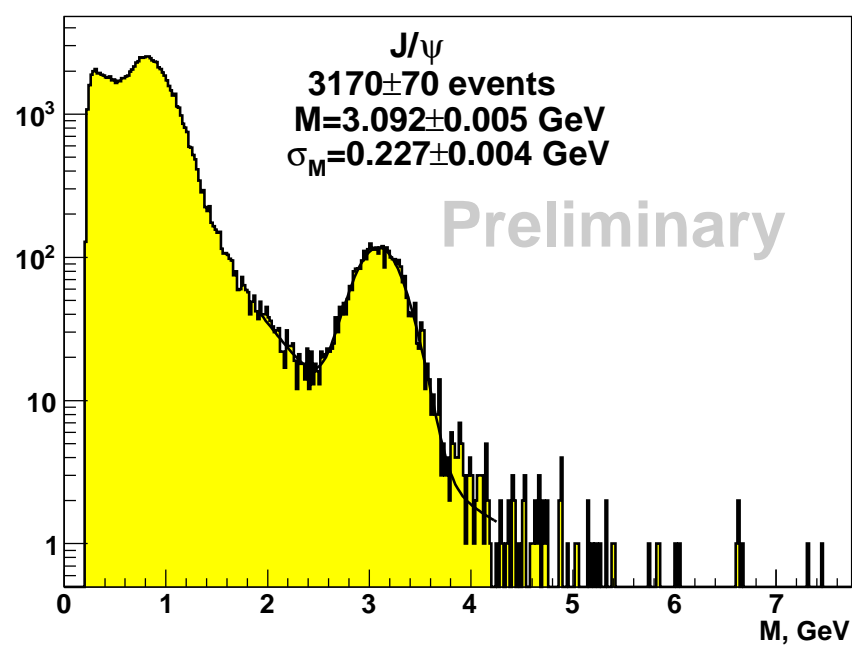

Figure 6: The measured $\mu^{+} \mu^{-}$invariant mass distribution. The number of events is obtained from the fit in the $J / \psi$ region.

consequence of the approximate chiral symmetry of QCD. Once the systematic corrections are worked out, ChPT can give firm predictions for the strong interaction dynamics of the Goldstone bosons (pions and kaons). The pion polarisabilities entail deviations from the pion Compton cross section within the Quantum Electro Dynamics (QED) expectation computed for structureless spin-0 particle.

The Primakoff process embedding the Compton scattering measured at COMPASS, is the following:

$$
\pi^{-} A \rightarrow \pi^{-} A \gamma
$$

where $A$ is the nucleus.

The differential cross section calculated in the Centre of Mass (CM) system can be written as the product of the differential cross section for a point-like particle times a term $P$ depending on the pion polarisabilities:

$$
\frac{d \sigma_{\pi \gamma}}{d \Omega_{C M}}=\left(\frac{d \sigma_{\pi \gamma}}{d \Omega_{C M}}\right)_{\text {point-like }} \cdot P
$$

where

$$
P=z_{-}^{2}\left(\alpha_{\pi}-\beta_{\pi}\right)+\frac{s^{2}}{m_{\pi}^{4}} z_{+}^{2}\left(\alpha_{\pi}+\beta_{\pi}\right)-\frac{\left(s-m_{\pi}^{2}\right)^{2}}{24 s} z_{-}^{3}\left(\alpha_{2}-\beta_{2}\right)
$$

$s$ is the Mandelstam variable, $z_{ \pm}=1 \pm \cos \theta_{C M}$ and $\theta_{C M}$ the scattering angle in the Centre of Mass (CM) system of the outgoing $\pi \gamma$ pair. The term $\left(\alpha_{2}-\beta_{2}\right)$ is the quadrupole polarisability difference. The effect of the difference of electric and magnetic polarisability $\left(\alpha_{\pi}-\beta_{\pi}\right)$ is strongest at backward angles, while the $\left(\alpha_{\pi}+\beta_{\pi}\right)$ influences the cross section mostly at large $\cos \theta$. The latter polarisability is expected to assume small values, but actually is enhanced in the cross section by the prefactor $\frac{s^{2}}{m_{\pi}^{4}}$. As a consequence, measuring the energy and angular dependence of the cross section allow for extraction of the two dipole polarisabilities separately.

A big theoretical and experimental effort was performed in order to extract the pion polarisabilities [8] with the ansatz that $\left(\alpha_{\pi}+\beta_{\pi}\right)=0$ and the results obtained so far disagree within each others and 
Table 1: Proposed running time, the respective total beam flux for pions and muons, and expected total errors on the pion polarisabilities (units $10^{-4} \mathrm{fm}^{3}$, except quadrupole polarisability value in units of $10^{-4} \mathrm{fm}^{5}$ ).

\begin{tabular}{|c|c|c|c|c|c|c|c|}
\hline Days & $\begin{array}{c}\pi \text { beam, } \\
\text { days }\end{array}$ & $\begin{array}{c}\mu \text { beam, } \\
\text { days }\end{array}$ & $\begin{array}{c}\text { Flux } \pi \\
10^{11}\end{array}$ & $\begin{array}{c}\text { Flux } \mu \\
10^{11}\end{array}$ & $\begin{array}{c}\alpha_{\pi}-\beta_{\pi} \\
\sigma_{\text {tot }}\end{array}$ & $\begin{array}{c}\alpha_{\pi}+\beta_{\pi} \\
\sigma_{\text {tot }}\end{array}$ & $\begin{array}{c}\alpha_{2}-\beta_{2} \\
\sigma_{\text {tot }}\end{array}$ \\
\hline \hline 120 & 90 & 30 & 59 & 12 & \pm 0.66 & \pm 0.025 & \pm 1.94 \\
\hline & & & & & & ChPT prediction & \\
\hline & & & & & 5.70 & 0.16 & 16 \\
\hline
\end{tabular}

partially with the ChPT predictions. An experimental clarification is needed and in COMPASS the pion polarisabilities can be extracted independently, namely without assumption $\left(\alpha_{\pi}+\beta_{\pi}\right)=0$.

In 2012 COMPASS II will start with the same spectrometer configuration as used in 2009 [2]. One of the key feature of the COMPASS experiment is the possibility to use the same spectrometer alternatively with two different beams: pion and muon beams. This allows the study of the systematic effects using a well known calibration process, namely the Primakoff scattering by muons.

In Table 1 the proposed running time, the respective total beam flux for pions and muons and the expected total errors on the pion polarisabilities are reported.

In addition to the pion polarisabilies measurement, Primakoff scattering with charged kaons can be observed for the first time and thus obtain the kaon polarisability. For 90 days of running and a total $K$ flux of $1.4 \times 10^{11}$ the error of $\left(\alpha_{\pi}-\beta_{\pi}\right)$ is expected to be $\pm 0.0810^{-4} \mathrm{fm}^{3}$ for a predicted ChPT value of $1.010^{-4} \mathrm{fm}^{3}$.

\section{Conclusion}

COMPASS is accomplishing its physics program by 2011 and in 2012 COMPASS II phase with a new physics program will start. The new measurements are mainly focused on the contribution of the intrinsic transverse quark momentum to the nucleon structure. DVCS and DVMP processes will give access to the GPDs in a wide $\mathrm{x}_{B j}$ region not explored by the past and near future experiments. The single polarised DY process will test the factorisation ansatz which predicts the sign change of the Sivers and Boer-Mulders functions between DY and SIDIS reactions. The major part of 2012 year will be devoted to the Primakoff measurements improving the accuracy of the pion polarisabilities and, due to the high data statistics collected, the kaon polarisability will be for the first time measured.

\section{References}

[1] P. Abbon et al.,Nucl.Instrum. Meth. A 577 (455) 2007.

[2] COMPASS Coll., COMPASS-II proposal, CERN-SPSC-201-014, SPSC-P-340, 17 May 2010.

[3] A. V. Belitsky et al.,Nucl. Phys. B 629 (323) 2002 .

[4] USQCD/UKQCD, Phys. Rev. Lett. 98 (051601) 2007

[5] A. Bacchetta, F. Conti, M. Radici, Phys. Rev. D 78 (074010) 2008

[6] M. Vanderhaeghen, Phys. Rev. D 60 (094017) 1999.

[7] K. Kumericki et al., arxiv:0904.0458.

[8] J. Friedrich, AIP Conf. Proc. Volume 1322 (457-462) Issue 1. 